\title{
Disordered critical wave functions in random-bond models in two dimensions: Random-lattice fermions at $E=0$ without doubling
}

\author{
Yasuhiro Hatsugai* \\ Department of Applied Physics, University of Tokyo, 7-3-1 Hongo Bunkyo-ku, Tokyo 113, Japan \\ Xiao-Gang Wen \\ Department of Physics, Massachusetts Institute of Technology, Cambridge, Massachusetts 02139 \\ Mahito Kohmoto \\ Institute for Solid State Physics, University of Tokyo, 7-22-1 Roppongi Minato-ku Tokyo 106, Japan
}

(Received 1 October 1996; revised manuscript received 10 March 1997)

\begin{abstract}
Random-bond Hamiltonians of the $\pi$ flux state on the square lattice are investigated. It has a special symmetry and all states are paired except the ones with zero energy. Because of this, there are always zero modes. The states near $E=0$ are described by massless Dirac fermions. For the zero mode, we can construct a random lattice fermion without a doubling and quite large systems (up to $801 \times 801$ ) are treated numerically. We clearly demonstrate that the zero mode is given by a critical wave function. Its multifractal behavior is also compared with the effective field theory. [S0163-1829(97)06628-9]
\end{abstract}

Random critical points in two dimensions appear in quantum Hall systems and in systems with spin-orbit coupling. Both critical points have important experimental implications, and both are in the strong coupling limit. Because of that we are still unable to calculate their critical exponents analytically, despite the efforts of more than a decade. Recently, a class of exactly solvable two-dimensional random critical points was found. ${ }^{1,2}$ However, it was pointed out that the critical points of Dirac fermion systems with random gauge fields contain an infinite number of relevant directions. ${ }^{2}$ Thus although the theory is exactly solvable at the critical point, it seems that it can never be realized in real or numerical systems. In this paper, however, we find that the $\pi$-flux states with bond randomness correspond to Dirac fermion systems with imaginary random gauge fields. Such critical points contain only two relevant directions and can be reached by fine tuning the two parameters. This result suggests that the exactly solvable random critical points in Dirac fermion systems probably can be realized in some random two-dimensional (2D) systems with Fermi points (such as graphite and 2D $d$-wave superconductors).

In this paper, we study random-bond models on the square lattice with only nearest-neighbor hopping. The models have $\pi$ flux per plaquette on the average. When all hopping matrix elements are real [in this case the model will be called the random hopping $(\mathrm{RH})$ model], we find that with weak randomness and in a continuum approximation, the states near zero energy are described by two copies of Dirac fermions coupled to imaginary random chemical potential, real random mass, and imaginary gauge fields. The results in Refs. 1 and 3 suggest that the continuum model has a line of random critical points, and due to the imaginary gauge fields and the imaginary chemical potential, the critical points have only one marginally relevant direction if we fix the energy to zero. Thus it could be possible to fine tune parameters to reach the critical line. In fact, the zero-energy states of the fine-tuned model are found to have a multifractal behavior and are critical. ${ }^{4}$ We also present direct numerical results indicating that the zero-energy states in the generic randombond model also appear to be critical with multifractal behaviors at least for the systems of sizes up to $801 \times 801$. The multifractal scaling function $f(\alpha)$ for the fine-tuned $\mathrm{RH}$ model is calculated numerically, which agrees with the exact result of the continuum theory. ${ }^{1,2}$ Another interesting point in our treatment is that we can avoid the fermion doubling as far as the zero-energy states are concerned.

In one dimension, some of the wave functions of the quasiperiodic systems show the critical behavior clearly. ${ }^{5}$ In two-dimensions, it was difficult to show the criticality clearly due to the limitation of available system sizes. However, since our models have several specialties as described below, we can treat quite large two-dimensional systems up to $801 \times 801$, which enables a clear demonstration.

The wave functions in a two-dimensional random system are believed to be always localized if the system has timereversal symmetry. Thus it is quite interesting that our models have random critical points. There are several reasons why the critical states are allowed in our models in two dimensions. Our random-bond model has a very special property that the random Hamiltonian anti-commute with the operator $\gamma$ defined below. This means that the eigenstates always appear in pairs with energies $E$ and $-E$. Therefore the states near zero energy are quite special, and one may wonder if they are critical states or not. We may also consider a pure system with a small diagonal hopping $m$ (which breaks the symmetry mentioned above). ${ }^{6}$ The symmetry is recovered by taking a limit $m \rightarrow 0$. When $m \neq 0$, there is an energy gap near $E=0$ and there are two energy bands. The Hall conductance of the lower band is $+\operatorname{sgn}(m)$ and $-\operatorname{sgn}(m)$ for the higher one. Since the nonzero Hall conductance is carried by extended states, there are paired extended states in the lower and the higher bands. By taking $m \rightarrow 0$, the paired extended states merge and disappear at $E=0$. This 
suggests the criticality of the $E=0$ states. Also from a point of the perturbation theory, the density of states of the pure state vanishes at zero energy. Therefore a usual treatment of the perturbative consideration has to be modified. This property, we believe, is also responsible for the appearance of the critical states at zero energy.

Our Hamiltonian is

$$
H=\sum_{\langle i, j\rangle} c_{i}^{\dagger} t_{i j} c_{j}+\text { H.c., }
$$

where the summation is over the nearest-neighbor bonds. We shall analyze the following two types of random-bond models near the $\pi$ flux: (i) random gauge model $t_{j+\hat{x}, j}=(-)^{j_{y}} e^{i \delta \theta_{x}(j)}, t_{j+\hat{y}, j}=e^{i \delta \theta_{y}(j)}$, and (ii) random hopping model, $t_{j+\hat{x}, j}=(-)^{j_{y}}+\delta t_{x}, t_{j+\hat{y}, j}=1+\delta t_{y}$. Here $\delta \theta_{x, y}(j)$ and $\delta t_{x, y}$ are real random variables. Numerical results of the wave functions for these two models are qualitatively similar. The model (i) belongs to the unitary ensembles. The model (ii) preserves time-reversal symmetry and belongs to the orthogonal ensembles, which is suitable for a comparison with the critical continuum theory.

In the absence of the randomness, the system is invariant under the translation and the Hamiltonian is written in momentum space:

$$
H_{0}=\int_{-\pi}^{\pi} \frac{d k_{x}}{2 \pi} \int_{0}^{\pi} \frac{d k_{y}}{2 \pi} \psi_{k}^{\dagger}\left(\begin{array}{cc}
\cos k_{y} & \cos k_{x} \\
\cos k_{x} & -\cos k_{y}
\end{array}\right) \psi_{k},
$$

with $\psi_{k}^{\dagger}=\left(c_{k}^{\dagger}, c_{k+(0, \pi)}^{\dagger}\right)$. We see that the zero-energy state is formed by the fermions with momentum $\boldsymbol{k}_{1}=(\pi / 2, \pi / 2)$ and $\boldsymbol{k}_{2}=(-\pi / 2, \pi / 2)$. Expanding it near $\boldsymbol{k}_{1}$ and $\boldsymbol{k}_{2}$, we find in the continuum approximation that $H_{0}$ becomes

$$
H_{0}=2 i \int d^{2} x \Psi^{\dagger}\left[\left(\begin{array}{ll}
\sigma_{1} & 0 \\
0 & -\sigma_{1}
\end{array}\right) \partial_{x}+\left(\begin{array}{ll}
\sigma_{3} & 0 \\
0 & \sigma_{3}
\end{array}\right) \partial_{y}\right] \Psi,
$$

with $\quad \Psi^{\dagger}=1 /\left(4 \pi^{2}\right) \int d \delta k_{x} \delta k_{y} e^{-i \delta k_{x} x-i \delta k_{y} y}\left(\psi_{k_{1}}^{\dagger}, \psi_{k_{2}}^{\dagger}\right) \quad$ and

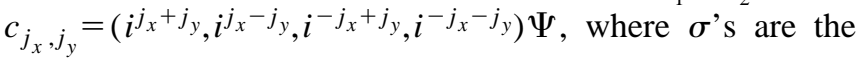
Pauli matrices. We assume that $\Psi$ take the same value on four sites $(x, y)=(2 m, 2 n), \quad(2 m+1,2 n), \quad(2 m, 2 n+1)$, $(2 m+1,2 n+1)$. Note that for weak impurities, the states near zero energy are described by the smooth function $\Psi(x)$. Therefore we can derive a continuum theory to describe those states near zero energy.

Before deriving the continuum theory with randomness, we first study the symmetries of the lattice Hamiltonian. Since the square lattice is bipartite, the transformation $c_{j_{x}, j_{y}} \rightarrow c_{j_{x}, j_{y}}^{\prime}=(-1)^{j_{x}+j_{y}} c_{j_{x}, j_{y}}$ induces sign changes of the Hamiltonian. It is expressed as $H(x) \rightarrow \gamma^{\dagger} H(x) \gamma=-H(x)$, $\gamma=\sigma_{1} \otimes \sigma_{1}$ where $H=\int d^{2} x \Psi(x)^{\dagger} H(x) \Psi(x)$ is the continuum Hamiltonian. The contribution from the randomness $\delta H \equiv H-H_{0}$ has a form $\delta H=\int d^{2} x \Psi^{\dagger} g(x) \Psi$ if we ignore the terms containing derivatives. The above condition implies that the most general form of the $g(x)$ is given by a linear combination of the 4 by 4 matrices: $\gamma_{1}=\sigma_{2} \otimes I, \quad \gamma_{2}=\sigma_{1} \otimes \sigma_{2}, \quad \gamma_{3}=-\sigma_{2} \otimes \sigma_{1}, \quad \gamma_{4}=I \otimes \sigma_{2}$ with coefficients $2 a_{i}(x), i=1, \ldots, 4$. The total Hamiltonian, after a change of basis to make $\gamma=-\sigma_{3} \otimes I\left(\Psi=T \Psi^{\prime}\right.$, $\left.T=\left(I \otimes I+i \sigma_{2} \otimes \sigma_{1}\right) / \sqrt{2}\right)$, is given by

$$
\begin{gathered}
H=\int d^{2} x \Psi^{\prime \dagger} 2\left(I \otimes \sigma_{1}\right)\left(\begin{array}{ll}
0 & D_{+} \\
D_{-} & 0
\end{array}\right) \Psi^{\prime}, \\
D_{ \pm}=i\left[\left(\partial_{x} \mp a_{1}\right) \sigma_{1} \mp\left(\partial_{y} \mp a_{2}\right) \sigma_{3}\right] \pm i a_{3} \mp \sigma_{2} a_{4} .
\end{gathered}
$$

Note that the averaged Hamiltonian is invariant under translation $(x, y) \rightarrow(x+1, y)$. This requires that $\left\langle a_{i}\right\rangle=0$ for $i=1,2,3$. The averaged Hamiltonian is also invariant under reflection $(x, y) \rightarrow(x,-y)$. This implies that $\left\langle a_{4}\right\rangle=0$.

The zero-energy state of $H$ satisfies

$$
D_{+} \psi_{+}=0, \quad D_{-} \psi_{-}=0,
$$

where $\left(\begin{array}{c}\psi_{-} \\ \psi_{+}\end{array}\right)=\Psi^{\prime}$. Thus the random-bond model contains two Dirac fermions in a continuum approximation, which is the famous fermion doubling of the lattice fermions. It causes several difficulties in numerical calculations. However, as we discuss below, we can avoid the doubling as far as the $E=0$ state is concerned.

Now let us discuss about the doubling that arises from the lattice models. Since our model is a nearest-neighbor hopping model on a bipartite lattice, we can write the Hamiltonian as

$$
H=\left(\left\{c_{+}^{\dagger}\right\},\left\{c_{-}^{\dagger}\right\}\right)\left(\begin{array}{ll}
O & \mathcal{D} \\
\mathcal{D}^{\dagger} & O
\end{array}\right)\left(\begin{array}{l}
\left\{c_{+}\right\} \\
\left\{c_{-}\right\}
\end{array}\right),
$$

where $\left\{c_{+}\right\}=\left\{c_{j_{x}, j_{y}} \mid j_{x}+j_{y}\right.$ is even $\}=\left\{c_{++}, c_{--}\right\}$is a set of fermion operators at one of the sublattices $(+)$ and $\left\{c_{-}\right\}=\left\{c_{j_{x}, j_{y}} \mid j_{x}+j_{y}\right.$ is odd $\}=\left\{c_{+-}, c_{-+}\right\}$is the other (-). Here let us assume a system to be $L_{x} \times L_{y}$ with both odd $L_{x}$ and $L_{y}$ with a fixed boundary condition. [We label the sites as $(1,1), \ldots,\left(L_{x}, L_{y}\right)$.] Then a total number of sites $L_{x} L_{y}=N_{+}+N_{-}$is odd with $N_{+}=N_{-}+1$ where $N_{+}=\#\{+$ sites $\}, \quad N_{-}=\#\{-$ sites $\}$. Therefore $\mathcal{D}$ is a $N_{+} \times N_{-}=N_{+} \times\left(N_{+}-1\right)$ rectangular matrix. The Schrödinger equation is reduced to $\mathcal{D D}^{\dagger} \phi_{+}=E^{2} \phi_{+}$and $\mathcal{D}^{\dagger} \mathcal{D} \phi_{-}=E^{2} \phi_{-}$. Any state with $E \neq 0$ has a pair state at $-E$ as ${ }^{t}\left(\phi_{+}, \phi_{-}\right)_{-E}={ }^{t}\left(\phi_{+},-\phi_{-}\right)_{E}$. Since $\operatorname{det}\left(\begin{array}{ll}O & \mathcal{D} \\ \mathcal{D}^{\dagger} & O\end{array}\right)=0$ (note that $\mathcal{D}$ is a form of $N_{+} \times N_{+}-1$ ), there is always zero mode. The wave function at $E=0$ satisfies

$$
\mathcal{D}^{\dagger} \phi_{+}^{E=0}=0, \quad \mathcal{D} \phi_{-}^{E=0}=0 .
$$

Since $\operatorname{dim} \phi_{+}^{E=0}=N_{+}$, the first one gives $N_{-}=N_{+}-1$ equations for $N_{+}$variables and there is a one -dimensional nonvanishing solution. (Therefore this zero mode is not degenerate in general.) On the other hand, since $\operatorname{dim} \phi_{-}^{E=0}=N_{-}=N_{+}-1$, the second one gives $N_{+}$equations for $N_{-}=N_{+}-1$ variables, this is overdetermined. Therefore we have $\phi_{-}^{E=0}=0$.

This gives an important restriction in the continuum model. It implies

$$
\psi_{-}=0
$$

in Eq. (6) since the transformation matrix between $|\Psi\rangle$ 's s and the site operators' $U T^{-1} U^{-1}$, only mixes operators within each sublattices. Therefore there is no degeneracy (doubling) for the $E=0$ state. This $E=0$ state is described by a usual two component spinor. Therefore the independent 


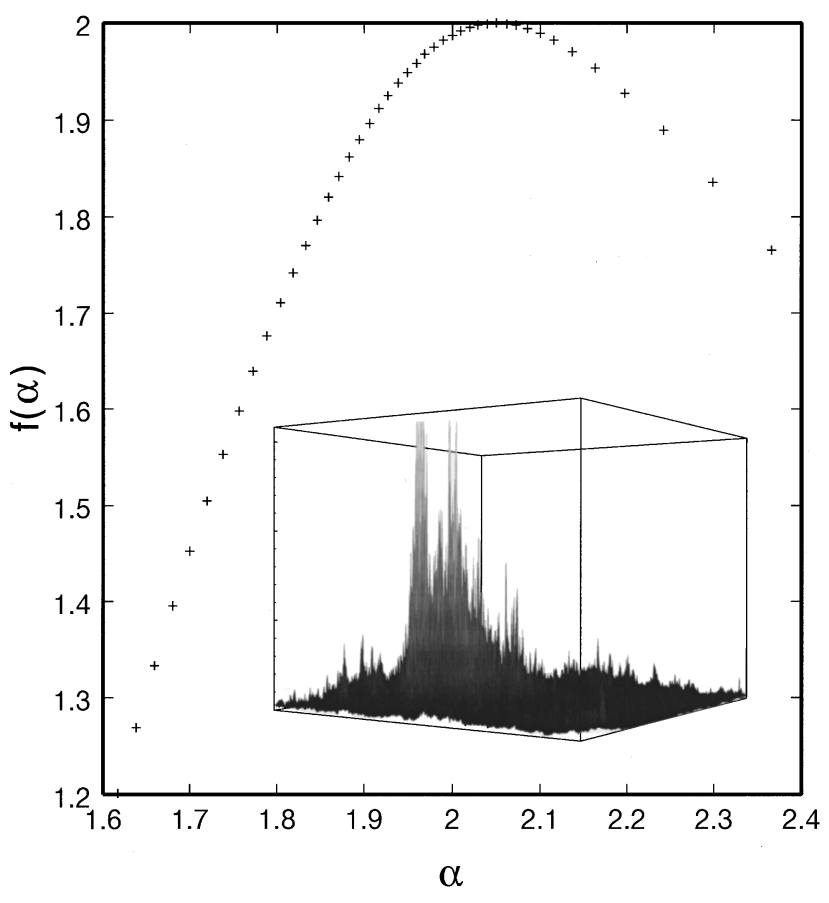

FIG. 1. The zero-mode wave function of the random gauge model and the corresponding $f(\alpha)$. The system size is $801 \times 801$ and $V=0.2$. The errors by the finite-size corrections in the box sizes is about the symbol sizes within the shown region but is larger in the other.

component is not four but two as far as the $E=0$ state is concerned. We could avoid the doubling by using the above special conditions.

Random gauge model. Now let us show numerical results for the random gauge model. In the previous numerical calculations for the $\pi$ flux state with randomness, ${ }^{7}$ it was not possible to treat large two-dimensional systems. In the present work, however, we only need the eigenstate of a semipositive definite operator $\mathcal{D D} \dagger$ with zero eigenvalue. This and the fact that the $E=0$ eigenstates are generally not degenerate enable us to treat large size systems up to $801 \times 801$. We take $\delta \theta_{x}(i)$ and $\delta \theta_{y}(i)$ to be uniform random numbers between $-V / 2$ to $V / 2$. In Fig. 1, the wave function is shown for $V=0.2$ with a system size of $801 \times 801$, where it is coarse grained over 4 sites on the plaquette. It seems to be neither localized nor extended in the usual manner. To understand the nature of the wave functions, the function $f(\alpha)$ obtained numerically is also shown. The box size dependence is carefully included in the calculations. ${ }^{5}$ The maximum of $f(\alpha)$ gives a Hausdorf dimension that is always two here since the wave functions are on the square lattice. The value $\alpha_{0}$, which gives the maximum of $f(\alpha)$, gives scaling of the dominant parts of the wave function. It is two for an extended state that gives uniform nonsingular scaling. For a critical state, $\alpha_{0} \neq 2$ and has a singular scaling. ${ }^{8,9}$ In our case, it apparently deviates from 2 , which means the zero mode is critical.

Random hopping model. The properties of random Dirac fermion models have been studied in detail recently. We study the random hopping model to make a comparison with the continuum theory. In the continuum approximation, the fact that $\delta t_{x, y}$ 's are real further requires $\gamma g(x) \gamma=g(x)^{*}$.
Thus we take the coefficients $a_{i}(x), i=1, \ldots, 4$ to be real. Note that $D_{ \pm}$happens to be the Hamiltonian of Dirac fermions with imaginary random gauge potentials $\left(a_{1}, a_{2}\right)$, an imaginary random chemical potential $a_{4}$, and a real random mass $a_{3}$. Let $\left(a_{1}, a_{2}\right), 2 a_{3}$, and $2 a_{4}$ have a Gaussian distribution with width $g_{A}, g_{V}$, and $g_{M}$, respectively. Averaging over randomness generates four-fermion interactions with $\left(g_{A}, g_{V}, g_{M}\right)$ as coupling constants. The one-loop $\beta$ functions for the above coupling constants are calculated in Ref. 3 as

$$
\begin{gathered}
\dot{g}_{A}=32 g_{M} g_{V}, \\
\dot{g}_{V}+\dot{g}_{M}=-8\left(g_{V}-g_{M}\right)^{2}, \\
\dot{g}_{V}-\dot{g}_{M}=-8\left(g_{V}+g_{M}\right)\left(g_{V}-g_{M}\right)-8 g_{A}\left(g_{V}-g_{M}\right) .
\end{gathered}
$$

[Note that compared to Ref. 3, we have an imaginary random chemical potential, a real random mass, and an imaginary random gauge potential. Thus the $\beta$ functions are modified accordingly with $\left(g_{A}, g_{V}, g_{M}\right) \rightarrow\left(-g_{A},-g_{V}, g_{M}\right)$.] The renormalization-group (RG) equation has a fixed line $\left(g_{A}, g_{V}, g_{M}\right)=\left(g_{A}, 0,0\right)$. Around the fixed line, $g_{V}-g_{M}$ is irrelevent (note $g_{A}>0$ ) and thus we may set $g_{V}=g_{M}$. In this case $g_{V}+g_{M}$ does not flow, and nonzero $g_{V}+g_{M}$ will drive $g_{A} \rightarrow \infty$. Thus the fixed line has at least one (marginally) relevent direction. Two-fermion operators are also relevant. But those two-fermion operators do not appear since $\left\langle a_{i}\right\rangle=0, i=1, \ldots, 4$ due to the discrete symmetries. Because of the imaginary random gauge potential, the results in Refs. 10 and 3 imply that all charge neutral operators are irrelevant, except two-fermion operators and four-fermion operators discussed above. Thus the fixed line has only one marginally relevent direction. It suggests that the RH model has a line of critical points at zero energy that is described by the line of critical points in the random Dirac fermion model. Those results further suggest that, once we fix the energy to be zero, there is only one marginally relevant direction for the critical line. Thus the critical line in the RH model contains two relevant directions. Due to its relation to the random Dirac fermion model, the critical line in the RH model is exactly solvable, and critical exponents can be calculated exactly.

To describe the critical line, set $a_{3}=a_{4}=0$. Let us assume the probability distribution of $\left[a_{1}(\mathbf{r}), a_{2}(\mathbf{r})\right]$ is given by $P$ $\propto \exp \left(-\int d^{2} r\left(1 / 2 g_{A}\right)\left[a_{1}^{2}(\mathbf{r})+a_{2}^{2}(\mathbf{r})\right]\right)$, where $g_{A}$, characterizing the strength of the gauge field fluctuations, is exactly marginal. Thus $g_{A}$ can be used to parametrize different critical points on the critical line. In general critical exponents are functions of $g_{A}$.

Note that the energy $E$ couples to an operator $\Psi_{+}^{\dagger} \Psi_{+}$. Since $\Psi_{+}$and $\Psi_{+}^{\dagger}$ belong to two independent Dirac fermion models described by $D_{+}$and $D_{-}$, respectively, the scaling dimension of $\Psi_{+}^{\dagger} \Psi_{+}$is simply the sum of the scaling dimensions of $\Psi_{+}$and $\Psi_{+}^{\dagger}$. From Ref. 10 , we see that the scaling dimensions of $\Psi_{+}$and $\Psi_{+}^{\dagger}$ are equal to $1 / 2$, which implies that the scaling dimension of $\Psi_{+}^{\dagger} \Psi_{+}$is 1 . This means that the dynamical exponent $z=1$ and the density of states scale as $N(E) \propto|E|$; both exponents are independent of $g_{A}$. 
In the continuum theory (5), the zero-energy state can be solved exactly. ${ }^{1}$ Introducing $\phi$ and $\chi$ through $a_{\mu}=\partial_{\mu} \phi+\epsilon_{\mu \nu} \partial_{\nu} \chi$, one can show that the zero-energy state (with, for example, $\gamma=1$ ) is given by

$$
\Psi_{+} \propto e^{i \phi+\chi}
$$

and the probability distribution of $\phi$ and $\chi$ is given by $P \propto \exp \left(-\int d^{2} r\left(1 / 2 g_{A}\right)\left\{[\partial \phi(\mathbf{r})]^{2}+[\partial \chi(\mathbf{r})]^{2}\right\}\right)$. The wave function (13) with this distribution was studied in detail in Refs. 1,2, and was shown to have a multifractal structure. The exact multifractal scaling function $f(\alpha)$ is defined on $d_{-} \leqslant \alpha \leqslant d_{+}$and is given by ${ }^{2}$

$$
f(\alpha)=8 \frac{\left(d_{+}-\alpha\right)\left(\alpha-d_{-}\right)}{\left(d_{+}-d_{-}\right)^{2}},
$$

where $\quad d_{ \pm}=2\left(1 \pm \sqrt{g_{A} / 2 \pi}\right)^{2} \quad$ for $\quad g_{A}<2 \pi \quad$ and $d_{+}=8 \sqrt{g_{A} / 2 \pi}, d_{-}=0$ for $g_{A}>2 \pi .{ }^{11}$ We see that for weak randomness $\left(g_{A}<2 \pi\right) f(\alpha)$ is peaked at

$$
\alpha_{0}=2+\frac{g_{A}}{\pi}
$$

The parameters $a_{i}(\mathbf{r})$ in the continuum model are directly related to the lattice randomness. We find that $a_{1}=-\Lambda \delta t_{j, j+\hat{x}}(-)^{j_{x}}, \quad a_{2}(j)=-\Lambda \delta t_{j, j+\hat{y}}(-)^{j_{x}+j_{y}}, \quad a_{3}(j)$ $=\Lambda \delta t_{j, j+\hat{x}}(-)^{j_{x}+j_{y}}$, and $a_{4}(j)=-\Lambda \delta t_{j, j+\hat{y}}(-)^{j_{y}}$ with a dimensional parameter $\Lambda$. Therefore if we chose $\delta t_{i j}$ to satisfy $\delta t_{j, j+\hat{x}}=\delta t_{j+\hat{y}, j+\hat{y}+\hat{x}}$ and $\delta t_{j, j+\hat{y}}=-\delta t_{j+\hat{x}, j+\hat{x}+\hat{y}}$, then $g_{V}=g_{M}=0$ and the lattice model will be on the critical line. We used this parametrization and performed a numerical calculation on the above fine-tuned $\mathrm{RH}$ model. We set $\delta t_{x, y}$ as Gaussian random numbers with a variance $V^{2}\left(g_{A}=\Lambda^{2} V^{2}\right)$. In Fig. 2, we have plotted a singular scaling function $f(\alpha)$. In the calculation, $g_{A}$ is obtained from a value of $\alpha_{0}$. The $f(\alpha)$ obtained from numerical calculation agrees well with the exact theoretical result Eq. (14).

In summary, we find that in the continuum approximation, the RH model at zero energy has a critical line with only one marginally relevent direction. This analytical result is supported by numerical calculations on the fine-tuned $\mathrm{RH}$ model. Therefore the RH model is described by the continuum random Dirac fermions as far as critical behavior is concerned. We also find that the zero-energy states of the

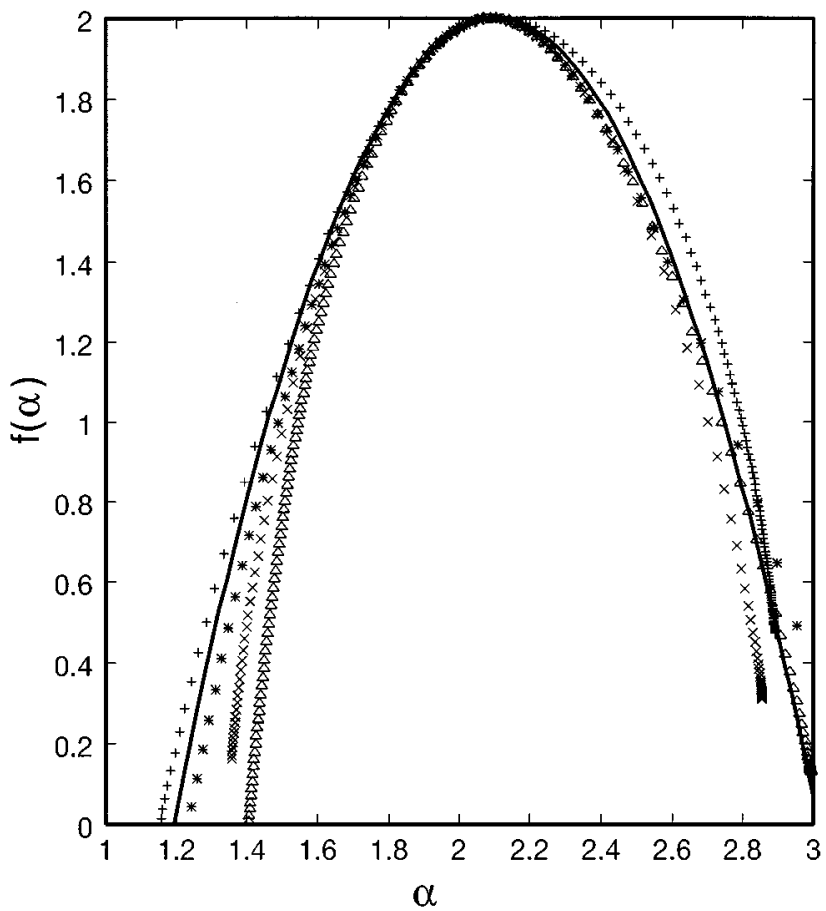

FIG. 2. Functions $f(\alpha)$ 's for the zero-mode wave functions of the RH model: $401 \times 401$ and $V=0.3$. The solid line is the analytical result. The different symbols are for the different randomness realizations.

general random bond models also appear to be critical according to our numerical results. However, the numerical calculations cannot eliminate the possibility of localization with huge correlation length (more than 800 lattice spacings), which may appear in our problem due to the marginally relevant direction.

This work was supported in part by Grant-in-Aid from the Ministry of Education, Science and Culture of Japan (Y.H., X.G.W., M.K.) and NSF Grant No. DMR-9411574 (X.G.W.). X.G.W. also acknowledges the support from the A.P. Sloan Foundation. The computation in this work has been partly done using the facilities of the Supercomputer Center, ISSP, University of Tokyo.
*Electronic address: hatsugai@coral.t.u-tokyo.ac.jp

${ }^{1}$ A. Ludwig, M. Fisher, R. Shankar, and G. Grinstein, Phys. Rev. B 50, 7526 (1994)

${ }^{2}$ C. de C. Chamon, C. Mudry, and X.G. Wen, Phys. Rev. Lett. 77, 4194 (1996).

${ }^{3}$ D. Bernard (unpublished).

${ }^{4}$ T.C. Halsey, M.H. Jensen, L. Kadanoff, I. Procaccia, and B. Shraiman, Phys. Rev. A 33, 1141 (1986).

${ }^{5}$ See H. Hiramoto and M. Kohmoto, Int. J. Mod. Phys. B 6, 281 (1992), and references therein.

${ }^{6}$ Y. Hatsugai and M. Kohmoto, Phys. Rev. B 42, 8282 (1990); J.H. Han, D.J. Thouless, H. Hiramoto, and M. Kohmoto, ibid. 50, 11 365 (1994); Y. Hatsugai, M. Kohmoto, and Y.-S. Wu, ibid. 54, 4898 (1996).

${ }^{7}$ Y. Hatsugai and P.A. Lee, Phys. Rev. B 48, 4204 (1993).
${ }^{8}$ B. Huckenstein, Rev. Mod. Phys. 67, 357 (1995).

${ }^{9}$ Y. Avishai, Y. Hatsugai, and M. Kohmoto, Phys. Rev. B 51, 13 419 (1995).

${ }^{10}$ C. de C.Chamon:, C. Mudry, and X.G. Wen, Nucl. Phys. B 466, 383 (1996); Phys. Rev. B 53, R7638 (1996); A.A. Nersesyan, A.M. Tsvelik, and F. Wenger, Phys. Rev. Lett. 72, 2628 (1994).

${ }^{11}$ The zero-energy wave function derived from the effective field theory of Ref. 2 has large amplitude fluctuations. It is also nonnormalizable in the thermodynamic limit, a fact which is central to the deviation of the parabolic $f(\alpha)$ in Eq. (14). The numerical wave functions for the zero modes of our random bond models shows similar large wave functions. The parabolic $f(\alpha)$ 's we find numerically provide evidence that these wave functions are both critical and non-normalizable in the thermo-dynamic limit, consistent with the effective field theory. 